POS $\quad$ PROCEEDINGS

\title{
PANDA Forward Spectrometer Calorimeter
}

\author{
P.A. Semenov* \\ IHEP NRC KI \\ E-mail: psemenov@ihep.ru
}

S.I. Bukreeva

IHEP NRC KI

ITEP NRC KI

S.K. Chernichenko

IHEP NRC KI

A.V. Sukhih

IHEP NRC KI

S. Diehl

University of Giessen

V. Dormenev

University of Giessen

R. Novotny

University of Giessen

V.V. Mochalov

IHEP NRC KI

D.A. Morozov

IHEP NRC KI

ITEP NRC KI

A.V. Ryazantsev

IHEP NRC KI

S.V. Ryzhikov

IHEP NRC KI

ITEP NRC KI

A.N. Vasiliev

IHEP NRC KI 
The PANDA experiment is one of the challenging projects being constructed on new FAIR facility near Darmstadt. It will use the antiproton beam from the High Energy Storage Ring colliding with an internal proton target and a general purpose spectrometer to carry out a rich and diversified hadrons physics program. One of the main PANDA detectors is a calorimeter complex consisting of a central PWO crystal based calorimeter and a forward shashlyk-type sampling calorimeter. The design of the PANDA Forward Spectrometer Calorimeter (FSC) is completed and validated by several test-beam measurements. FSC Technical Design Report recently was approved by FAIR experts committee and construction of the detector is expected to be started in the next year. The detector production-ready status including mass-production techniques developed at IHEP, Protvino is presented in the report.

54th International Winter Meeting on Nuclear Physics 25-29 January 2016

Bormio, Italy

*Speaker. 


\section{Introduction}

Facility for Antiproton and Ion Research (FAIR) is a new unique accelerator complex under construction at GSI site near Darmstadt, Germany. One of the four experimental programs here is a universal detector for strong interaction studies in proton-antiproton annihilations -PANDA. PANDA will be installed at High Energy Storage Ring (HESR), one of the FAIR accelerator complex rings.

One of the unique features of the HESR is a possibility to provide beam for PANDA in two operating modes. The first one is high-luminosity mode with up to $10^{11}$ particles in the ring (peak luminosity up to $2 \cdot 10^{32} \mathrm{~cm}^{-2} \mathrm{~s}^{-1}$ ). The second operation mode is the high-resolution mode with up to $10^{10}$ particles in the ring (peak luminosity up to $2 \cdot 10^{31} \mathrm{~cm}^{-2} \mathrm{~s}^{-1}$ ) and a momentum spread down to a few times $10^{-5}$.

The aim of the PANDA versatile experimental program is to answer burning questions in the field of Quantum Chromo Dynamics (QCD). In contrast to collider experiments as CMS, ATLAS or LHCb at CERN with proton-proton collisions, or CDF and D0 at Fermilab, USA, with antiprotonproton collisions, the emphasis is not put on highest centre-of-mass (c.m.) energies for studies at the energy frontier. PANDA was designed for very high precision measurements in the lower c.m. energy regime between 2.3 and $5.5 \mathrm{GeV}$. The main objectives of the PANDA detector design are the full geometrical acceptance, high resolution for tracking, particle identification and calorimetry, high rate capabilities, and a versatile readout providing effcient event selection. To obtain a good momentum resolution, the detector is split into a Target Spectrometer housed in a superconducting solenoid magnet surrounding the interaction point and a Forward Spectrometer employing a dipole magnet for the momentum analysis of charged small-angle tracks. In the Target Spectrometer high-precision electromagnetic calorimetry is required over a large range from a few $\mathrm{MeV}$ up to several $\mathrm{GeV}$ in energy deposition. Lead-tungstate (PWO) is chosen as scintillating crystal for the calorimeters in the Target Spectrometer because of its high density, fast response, and good light yield, enabling high energy resolution and a compact calorimeter configuration [1]. The crystal calorimeter is complemented in the Forward Spectrometer with a shashlyk-type sampling calorimeter consisting of 378 modules each made from 4 cells of $55 \times 55 \mathrm{~mm}^{2}$ size covering about $3 \mathrm{~m}$ by $1.5 \mathrm{~m}$.

\section{Forward Shashlyk Calorimeter}

Sampling calorimeters, constructed from alternating layers of organic scintillator and heavy absorber materials, have been used in high energy physics since tens of years. The sampling fraction of such calorimeters, i.e. the thickness ratio of active (scintillator) and passive (absorber) layers determines the lateral size of the electromagnetic showers. The thickness of the scintillator plates effects the light yield and, in turn, determines the stochastic term of the energy resolution. Therefore, the choice of the calorimeter sampling is based on the compromise between energy resolution and the lateral shower size. In a shashlyk-type sampling calorimeter the scintillation light is absorbed, re-emitted and transported to a photo detector by a wavelength shifting (WLS) optical fibers running through the calorimeter module longitudinally (along the beam direction). In the past, the typical stochastic term of the energy resolution was about $10 \%$ for large electromagnetic 
calorimeters of sampling type. However, the energy resolution can be significantly improved using finer sampling ratio. PANDA Forward Shashlyk Calorimeter (FSC) prototypes, manufactured at IHEP, Protvino, showed stochastic term of the energy resolution about 3\% over wide energy range from $50 \mathrm{MeV}$ up to $19 \mathrm{GeV}$.

The concept of the FSC design is based on the existing experience with the KOPIO project at BNL, USA [2, 3].

The structure of the detector is composed of lead plates as passive absorber and organic scintillator tiles as active elements. The main mechanical properties of the Panda FSC are compiled in Table 1.

\begin{tabular}{lcc}
\hline Title & Value & Units \\
\hline Overall detector width (x direction) & 4.9 & $\mathrm{~m}$ \\
Overall detector height (y direction) & 2.2 & $\mathrm{~m}$ \\
Overall detector depth (z direction) & 1150 & $\mathrm{~mm}$ \\
Overall detector weight & 14.7 & $\mathrm{tons}$ \\
Number of channels (including beam pipe zone) & 1512 & $\mathrm{pcs}$ \\
Number of modules (including beam pipe zone) & 378 & $\mathrm{pcs}$ \\
Module weight & 21 & $\mathrm{~kg}$ \\
Module cross section & $110 \times 110$ & $\mathrm{~mm}^{2}$ \\
Cell cross section & $55 \times 55$ & $\mathrm{~mm}^{2}$ \\
Scintillator tile thickness & 1.5 & $\mathrm{~mm}$ \\
Lead plate thickness & 0.275 & $\mathrm{~mm}$ \\
Beam pipe zone & $9=(3 \times 3)$ & $\mathrm{modules}$ \\
\hline
\end{tabular}

Table 1: Main mechanical properties of the FSC.

General detector view and design features are shown in the Figure 1. The figure shows the PANDA Forward Spectrometer Calorimeter placed on the Forward Spectrometer support. The region of a single module is zoomed, allowing a view into the calorimeter structure with the sandwich of scintillator and lead tiles and the bunches of traversing WLS fibres, funneled to photo detectors, one for each of the four cells of one module. The "LEGO"-type locks used to firmly join the tiles are zoomed even more.

\section{Test beam results}

Several FSC prototypes have been built at IHEP to test various configurations and satisfy requirements for energy resolution, light collection uniformity and low registration energy threshold. FSC should register photon in wide energy range up to $15 \mathrm{GeV}$. Therefore, prototypes performance was studied both at high energy region up $19 \mathrm{GeV}$ electrons at IHEP and at low region down to 50 $\mathrm{MeV}$ photons at MAMI. Energy resolution for high energy electrons and low energy photons are shown in the Figure 2 and Figure 3 below. Stochastic term is equal to $3.15 \%$ for energy resolution in the high energy region and $4.2 \%$ in the low energy region.

Position resolution depends strongly on the position of the shower vertex - close to the center of the cell the resolution is worse. At the center of the cell we measured $3 \mathrm{~mm}$ (sigma) resolution 


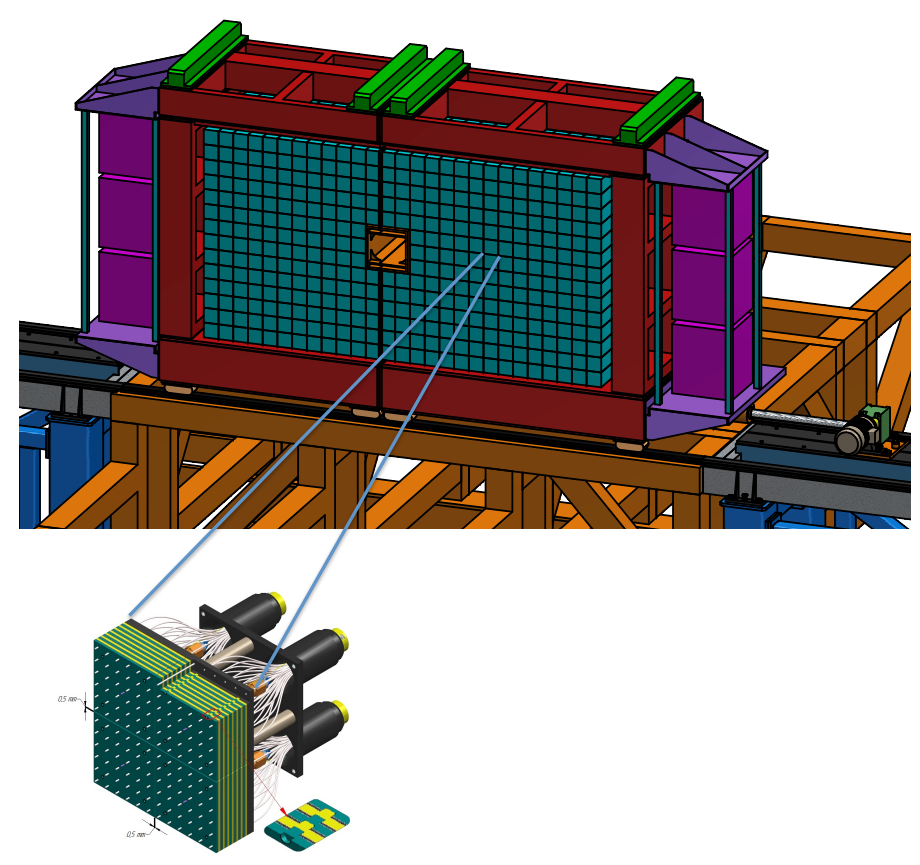

Figure 1: FSC general view and zoomed internal structure of the FSC module.

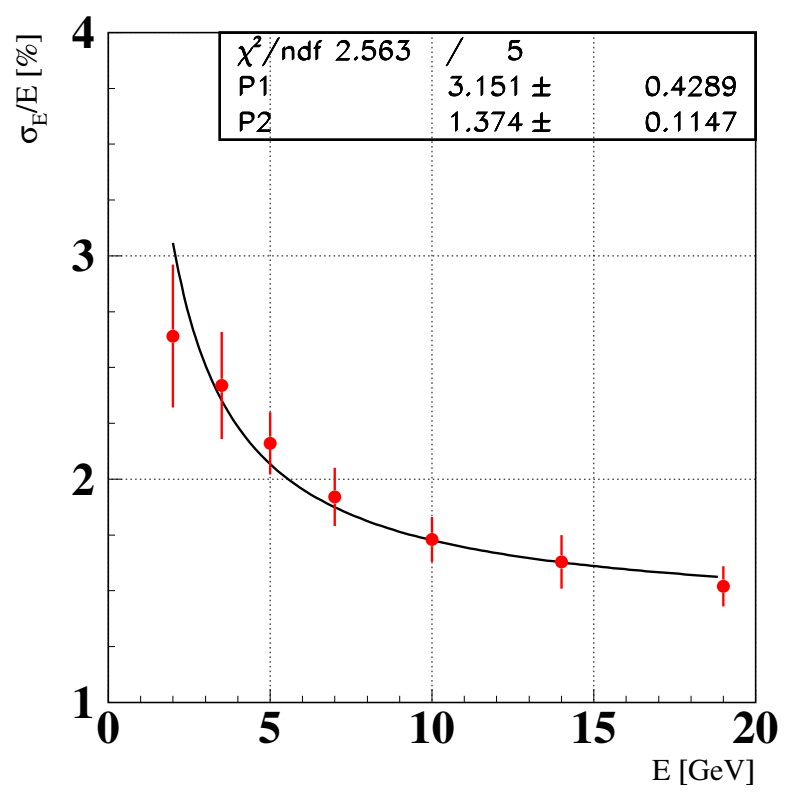

Figure 2: FSC prototype energy resolution dependence on energy for high energy region up to $19 \mathrm{GeV}$. 


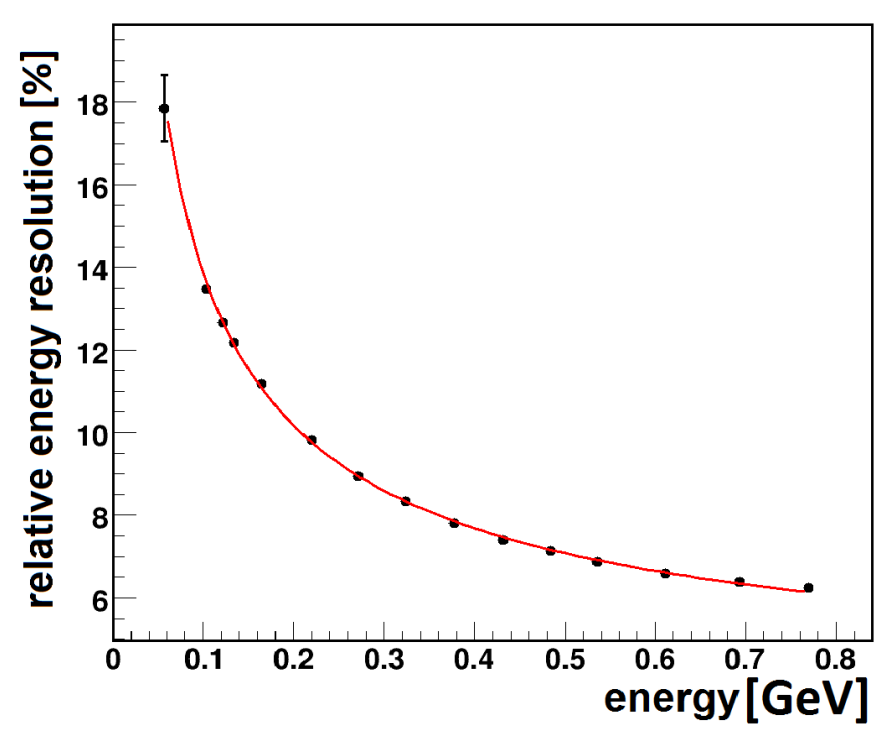

Figure 3: FSC prototype energy resolution dependence on energy for low energy region below $1 \mathrm{GeV}$.

at $19 \mathrm{GeV}$ and $15 \mathrm{~mm}$ at $400 \mathrm{MeV}$. While at the edge of the cell the resolution was $1.5 \mathrm{~mm}$ and 8 mm correspondingly.

Also time resolution was measured comparing time of the incoming signals from two neighboring cells. The stochastic term appeared to be about $100 \mathrm{ps}$ (see Figure 4), which is important to have since PANDA will not use hardware trigger to start event digitization.

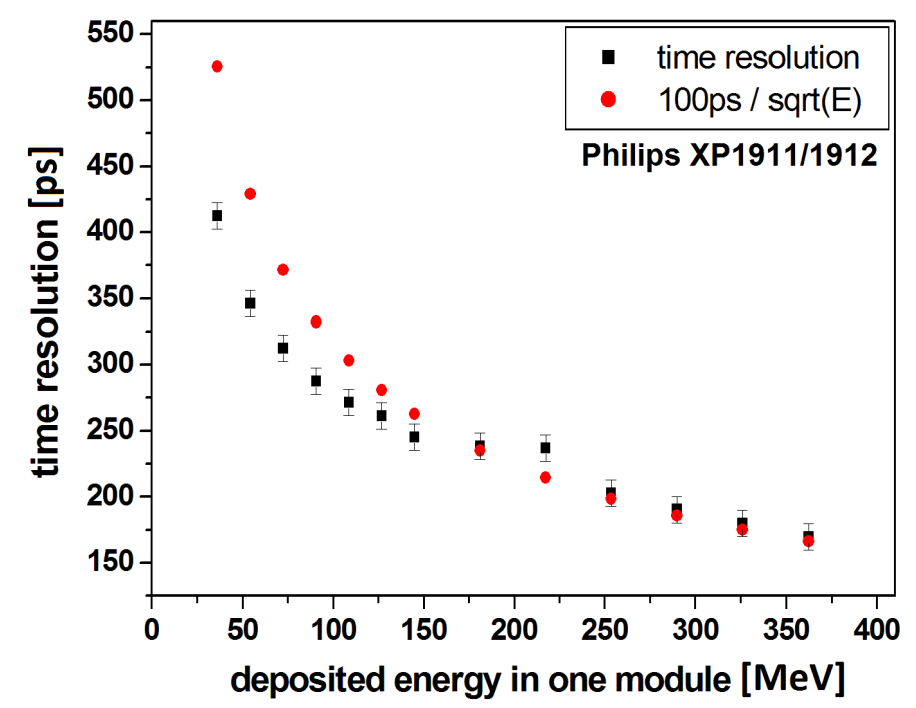

Figure 4: Time resolution of a single cell as a function of deposited shower energy. The red dots correspond to a resolution of $100 \mathrm{ps} / \sqrt{E / G e V}$ with $\mathrm{E}$ given in $\mathrm{GeV}$. 


\section{Design of the FSC detector}

The FSC detector is designed in planar geometry, covering the most forward angular range up to $5^{\circ}$ in the vertical and $10^{\circ}$ in the horizontal direction. The overall dimensions of the detector frame are $3.6 \mathrm{~m}$ in width and $2.2 \mathrm{~m}$ in height.

The active volume of the FSC consists of $54 \times 28$ cells or 378 modules. Each module has four optically isolated cells. Cell sizes are $55 \mathrm{~mm}$ x $55 \mathrm{~mm}$. Scintillator tiles thickness is $1.5 \mathrm{~mm}$, lead plate is 275 microns thick. 380 layers of the scintillator and lead resulted in 20 radiation length module longitudinal size. The light collection provided by 18 WLS optical fiber loops (Kuraray Y11 $1 \mathrm{~mm}$ in diameter) for each cell. Each fiber loop goes though the cell and bends at the front side of the module, collecting light and transporting it to the photomultiplier tube (PMT, Hamamatsu R7899) (Figure 5). To prevent a light leakage each tile edge is covered by a thick layer of white paint.

The gain of the PMT is monitored by the LED based high precision, stable monitoring system. Monitoring system ligh pulse from a single powerfull LED distributed to each module by means of optical fibers, connected to the module from the back (PMT) side, as shown in the Figure 6. Light from the fiber goes through the optical connector and divided into four fibers to monitor each PMT gain.

One of the features of the FSC scintillator tiles are LEGO-type pins and pits for a precise (20 microns tolerance) relative position of the tiles (Figure 7). Besides the relative positioning of the tiles, LEGO-type pins are important to provide an air gap between tiles to improve light collection.

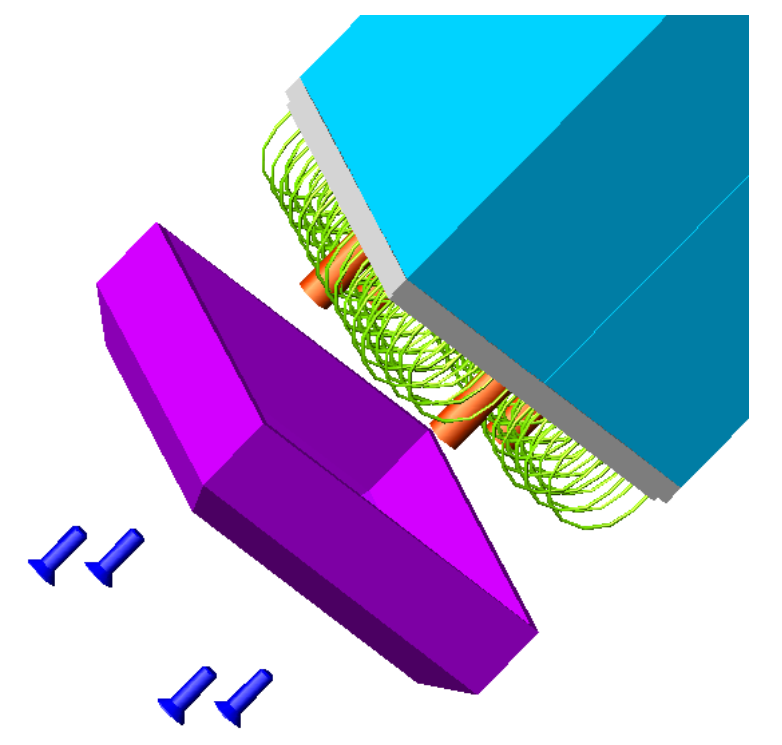

Figure 5: A front-side view of the FSC module showing the front cover, the WLS fibre loops, and the pressure plate.

High voltage for the PMT is supplied by the HV generators of Cockroft-Walton type. The combination of the PMT and Cockroft-Walton type bases is important to satisfy the requirements 


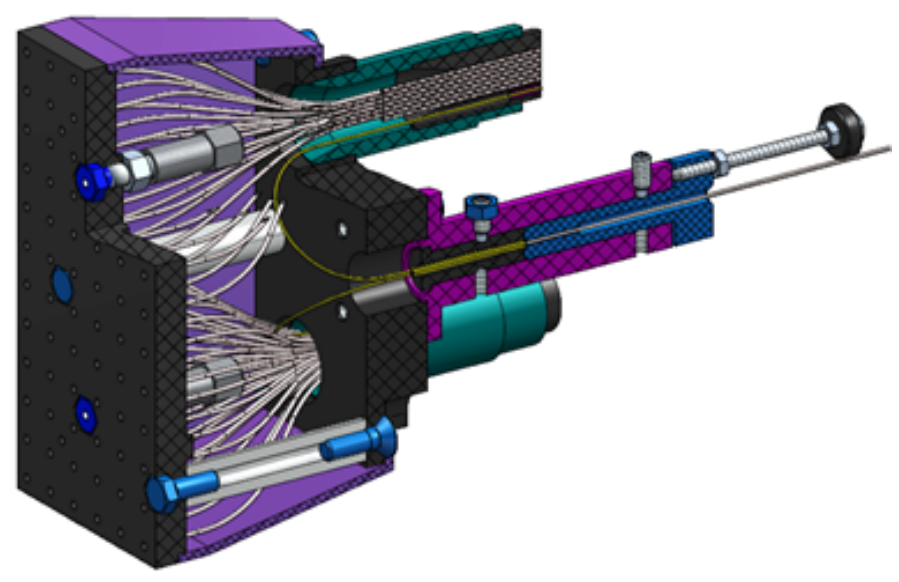

Figure 6: Sketch of the 3D view of the assembled back-side region of the shashlyk module with lightmonitoring system connector.

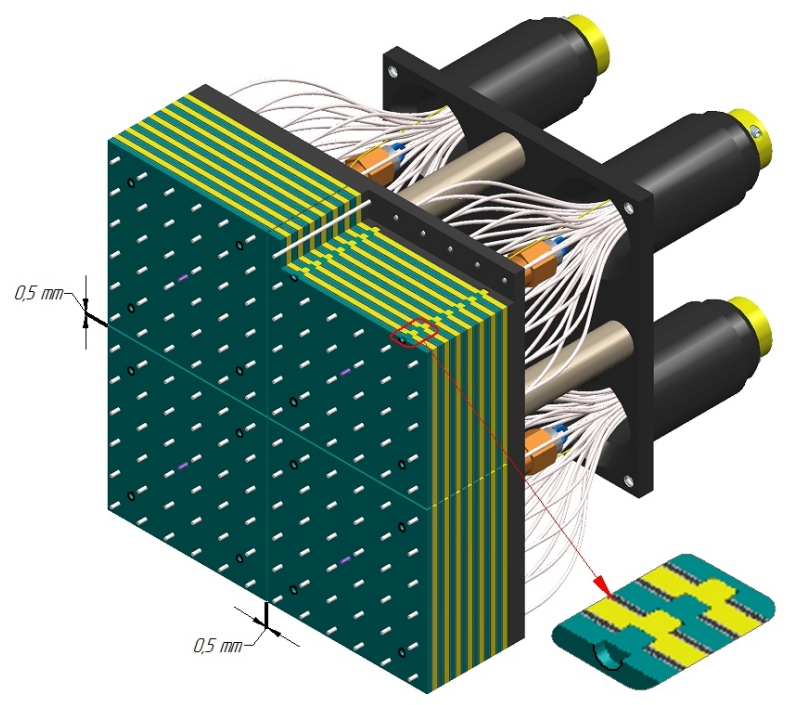

Figure 7: 3D view of the stack of scintillator tiles locked by LEGO-type pins and holes.

of high rate (up to $1 \mathrm{MHz}$ per cell) signals detection capability of the FSC.

\section{Manufacturing of the FSC modules}

The technology of the mass production of shashlyk modules is developed at IHEP. Scintillator workshop of IHEP has molding machines to produce tiles with required configuration, squeezers to punch lead plates, assembly tooling and experienced personnel (Figures 8,9,10).

During prototype production it took a day to assemble one four-cells module. This means the whole FSC detector for PANDA experiment can be produced at IHEP within a couple of years. 


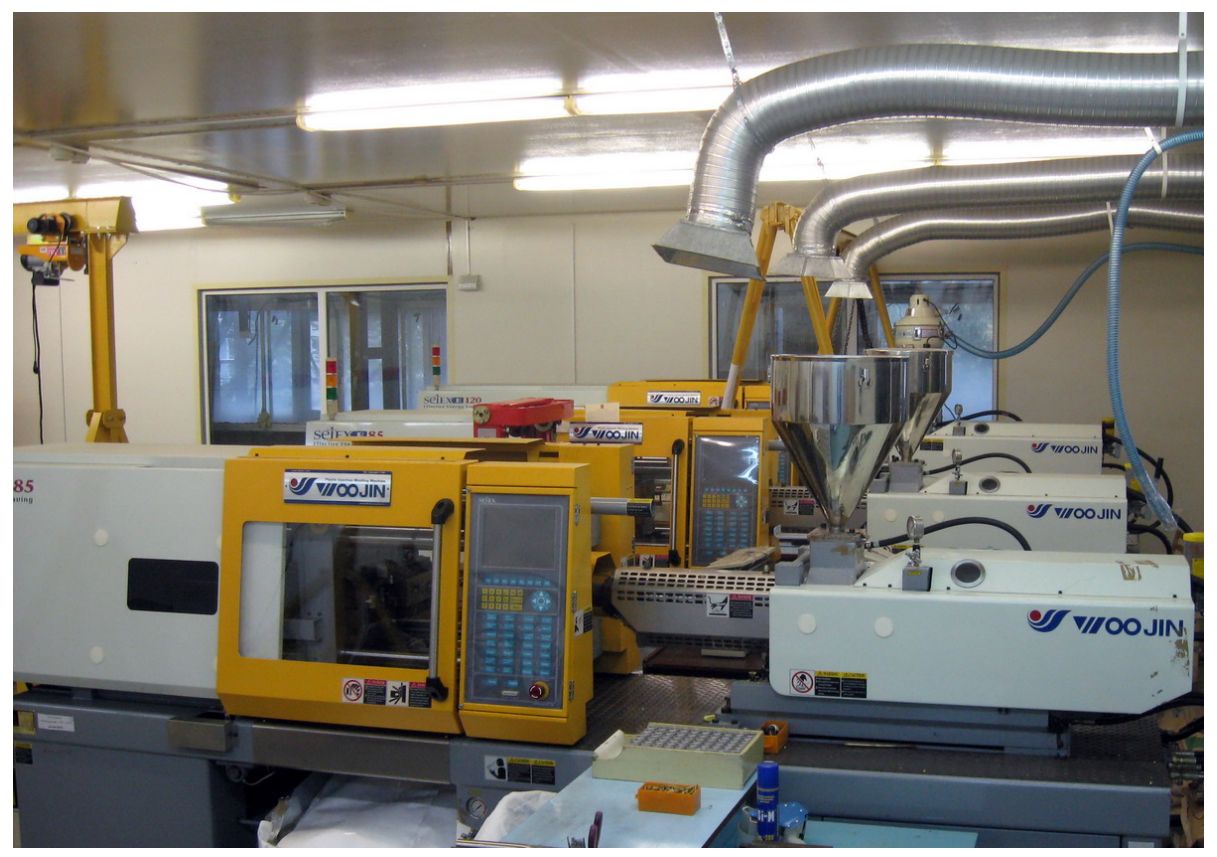

Figure 8: High pressure molding machines installed at IHEP scintillator department.

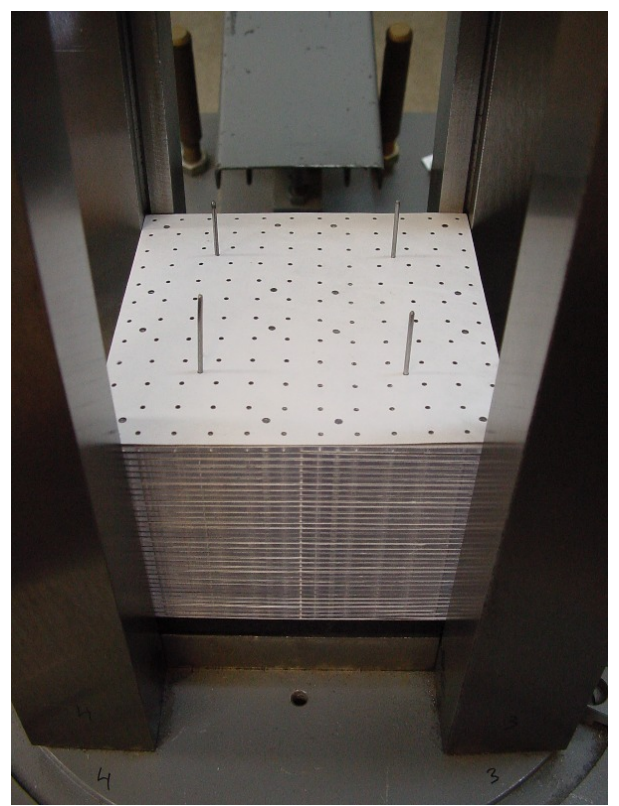

Figure 9: FSC module assembly tool. To put the fibers ( $1 \mathrm{~mm}$ diameter) through the module the tiles should be precisely positioned relative to each other. 


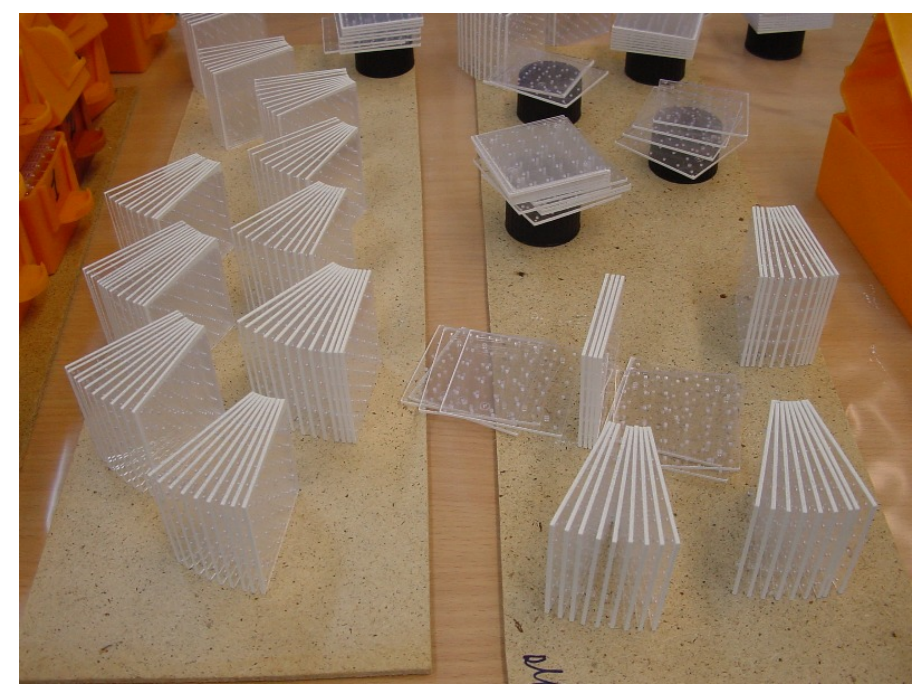

Figure 10: Molded scintillating tiles during the procedure of edges treatment.

FSC Technical Design Report was approved at the begining of 2016.

\section{Acknowledgments}

The work was carried out with the financial support of FAIR-Russia Research Center.

\section{References}

[1] W. Erni et al., PANDA Collaboration, Technical Design Report for PANDA Electromagnetic Calorimeter, arXiv:0810.1216 [physics.ins-det], 2008.

[2] G. Atoyan et al., Nucl. Instr. and. Meth. A 584, 291, 2008.

[3] G. Atoyan et al., Test beam study of the KOPIO Shashlyk calorimeter prototype, Proceedings of "CALOR 2004", May 2004. 